\title{
CONDIÇÕES DE TRABALHO E SAÚDE DOS MÉDICOS EM SALVADOR, BRASIL
}

\author{
Carlito lopes Nascimento Sobrinho*, fernando Martins Carvalho, tárcyo Antonio Silva Bonfim, Carlos Adriano Souza Cirino, \\ Isis Sacramento Ferreira \\ Trabalho realizado no Departamento de Saúde, Universidade Estadual de Feira de Santana, e no Departamento de Medicina Preventiva, \\ Faculdade de Medicina, Universidade Federal da Bahia.
}

${ }^{*}$ Correspondência:

BR 116, Km 03, Campus

Universitário, Feira de

Santana, Bahia,

Cep: $44031-460$

Tel/fax (75) 3224-8093

lua@uefs.br

\begin{abstract}
RESUMO
Oвjetrvos. Descrever as condições de trabalho e de saúde dos médicos da cidade de Salvador, Bahia, Brasil.

Métodos. Utilizando delineamento transversal, estudou-se uma amostra aleatória de 350 médicos. Num questionário individual, autoaplicável, enviado pelo correio, foram coletadas informações sobre a saúde física, mental, hábitos de vida e condições de trabalho dos médicos.

REsultados. Os médicos referiram elevada sobrecarga de trabalho, excesso de trabalho em regime de plantão, múltiplas inserções, contratação precária sob a forma de remuneração por procedimento e baixa remuneração. A atividade de consultório particular foi referida por 45,2\% dos médicos, mas apenas I,5\% exercia exclusivamente esta atividade. As queixas de saúde mais freqüentes estavam relacionadas à postura corporal e à saúde mental. A prevalência de distúrbios psíquicos menores, medida pelo Self Report Questionnaire (SRQ-20), foi de 26\%.
\end{abstract}

Conclusäo. As condições de trabalho e saúde observadas apontam para a necessidade de mudanças profundas na organizaçãa do trabalho médico.

UnITERMOS: Trabalho. Saúde. Médicos.

\section{INTRODUÇão}

O sistema de saúde no Brasil passa por uma profunda crise em seus componentes público e privado, a qual afeta a população em geral, os profissionais de saúde e os médicos, em particular. Os médicos queixam-se da remuneração, das condições de trabalho, dos processos de trabalho, dificuldade de reciclagem profissional, gerando pessimismo e desestímulo em relação ao futuro da profissão e do sistema de saúde $e^{1,2,3}$.

Em 1995 e 2003, realizaram-se amplas pesquisas de âmbito nacional, retratando as mais recentes e principais características dos médicos e de seu mercado de trabalho. Destacaram-se a crescente participação do sexo feminino na categoria, concentração de profissionais nos centros urbanos, principalmente nas grandes cidades, ritmo intenso de trabalho, jornadas de trabalho prolongadas, predomínio das atividades de plantão, principalmente entre os médicos mais jovens, sobrecarga de trabalho, insatisfação com a remuneração e com a profissão $0^{3,4}$.

Em estudo sobre o mercado de trabalho médico no Estado de São Paulo, observou-se que a maior parcela dos médicos aliava trabalho assalariado e prática autônoma em consultórios e organizações hospitalares, numa jornada que chegava a acumular três ou mais atividades. No setor público, predominava a contratação de médicos de forma assalariada e, no setor privado, por prestação de serviços por meio de cooperativas ou empresas médicas. Esta forma de contratação foi considerada como uma tendência no mercado, principalmente nos hospitais privados lucrativos ${ }^{5}$.

Existem poucos estudos sobre as condições de saúde dos médicos no Brasil, predominando os que enfocam a saúde mental desses trabalhadores $6,7,8,9,10,11,12$. Mesmo nos países desenvolvidos, poucos são os estudos que buscam associar as condições de trabalho e saúde dos médicos e também destacam-se os estudos sobre a prevalência de transtornos mentais, uso de drogas e suicídios entre médicos ${ }^{13,14,15,16,17,18,19}$.

Este trabalho objetivou descrever as condições de trabalho e os principais problemas de saúde referidos pelos médicos de Salvador, Bahia.

\section{Métodos}

Realizou-se estudo epidemiológico de coorte transversal, com uma amostra aleatória dos médicos que residiam na cidade do Salvador, registrados no Conselho Regional de Medicina do Estado da Bahia. O tamanho da amostra foi calculado considerando-se um total de 7.897 médicos, com nível de confiança de $95 \%$, precisão absoluta de $5 \%$, proporção esperada para o evento estudado de $50 \%$, resultando num tamanho amostral igual a 38420. Decidiu-se estudar até 800 médicos para compensar possíveis perdas e recusas ${ }^{21,22}$.

Para a coleta de dados, utilizou-se um questionário padronizado, respondido pelos próprios médicos, não sendo necessária identificação. O questionário tinha cinco blocos de questões: $1^{\circ}$ bloco: identificação geral do entrevistado (destinado a caracterizar os indivíduos integrantes da amostra segundo sexo, idade, especialização, tempo de trabalho profissional, carga horária total trabalhada/semana, turnos de trabalho etc.); $2^{\circ}$ bloco: características do ambiente de trabalho, percebidas pelo médico como nocivas à saúde; $3^{\circ}$ bloco: queixas de doenças (para avaliar a situação global de saúde dos indivíduos, buscando identificar queixas e sintomas de alguns agravos à saúde, tais como, distúrbios do sono, distúrbios auditivos e visuais, alergias, infecções 
recorrentes, distúrbios gastrintestinais, dentre outros problemas de saúde mais freqüentes); $4^{\circ}$ bloco: avaliação da saúde mental dos trabalhadores por meio de um instrumento de detecção de distúrbios psíquicos menores (DPM), o Self-Report Questionnaire(SRQ-20), e um instrumento de triagem de alcoolismo, o Cage; $5^{\circ}$ bloco: questões sobre doenças e acidentes de trabalho, problemas de saúde recentes e hábitos de vida.

O Self Report Questionnaire (SRQ-20), foi projetado para uso em estudos de morbidade psiquiátrica em instituições de cuidados primários de saúde em países em desenvolvimento ${ }^{23,24}$. O grau de suspeição para distúrbios psíquicos foi avaliado a partir do escore alcançado por cada médico(a) no SRQ-20. A avaliação adotou o ponto de coorte igual ou maior que sete respostas positivas, já validado em outras pesquisas ocupacionais, quando foram obtidos valores aceitáveis de sensibilidade, especificidade e valores preditivos ${ }^{25,26,27,28,29}$.

$\bigcirc$ Cage constitui-se de quatro questões que têm por objetivo identificar suspeitos de alcoolismo. O termo Cage é originário das palavras-chaves incluídas em cada uma das quatro questões em inglês, respectivamente: cut-down, annoyed, guilty e eye-opener. O Cage é utilizado como teste de triagem para detecção de abuso no consumo de bebidas alcóolicas, adotando-se o ponto de coorte em duas ou mais respostas positivas para as quatro questões do teste. Apresenta alta sensibilidade, especificidade e valores preditivos tanto na sua versão em inglês como na versão em português ${ }^{30}$.

Os questionários foram acompanhados de carta de apresentação e justificativa do trabalho, referendados pelas entidades representativas da categoria médica no estado da Bahia (Conselho Regional de Medicina do Estado da Bahia, Sindicato dos Médicos do Estado da Bahia e Associação Bahiana de Medicina). Os questionários foram enviados pelo correio, com retorno pago, após ampla campanha de divulgação do estudo, realizada em diversos veículos de comunicação (jornais escritos, programas de televisão, rádios $A M$ e FM) e divulgação direta em clínicas e hospitais, por meio de cartazes e folhetos. Além disso, os médicos selecionados na amostra foram contatados por telefone, buscando minimizar perdas e recusas. A coleta de dados ocorreu de agosto de 2002 a março de 2003. Os médicos que não devolveram os questionários pelo correio foram contatados por telefone, sendo solicitado o retorno do questionário. Um termo de consentimento livre e esclarecido foi enviado junto com o questionário, sendo garantido o sigilo das informações. 0 estudo foi aprovado pelo Comitê de Ética em Pesquisa do Hospital Universitário Professor Edgard Santos, da Universidade Federal da Bahia, seguindo assim as recomendações formais ${ }^{31}$.

A análise estatística dos dados foi feita no programa SPSS for Windows $^{32}$

\section{Resultados}

Estudou-se 350 médicos (43,7\% da amostra total). Entre os 800 médicos originalmente elegíveis, houve I 20 (I5\%) perdas, caracterizadas pelo endereço desatualizado ou errado e inexistência de telefones para contato. Os $330(42,3 \%)$ restantes foram considerados como recusas, pois foram contatados pelo correio e por telefone, mas não devolveram os questionários enviados.

Dos médicos estudados, $54,4 \%$ eram do sexo masculino e

\begin{tabular}{lcccc}
\hline \multicolumn{5}{c}{ Tabela I - Características sociodemográficas da amostra de } \\
médicos, Salvador, Bahia, 2002 \\
\hline \multirow{4}{*}{ Sexo } & Masculino & $\frac{\mathbf{n}}{190}$ & $\frac{\%}{54,4}$ & $\frac{\mathbf{N}^{*}}{349}$ \\
Faixa etária & Feminino & 159 & 45,6 & \\
& $<45$ anos & 184 & 53,2 & 346 \\
Situação conjugal & t45 anos & 162 & 46,8 & \\
& Casado & 199 & 57,2 & 348 \\
Naturalidade & Solteiro & 76 & 21,8 & \\
& Outros & 73 & 21,0 & \\
& Salvador & 181 & 52,0 & 348 \\
Linhagem médica & Interior da Bahia & 120 & 34,5 & \\
& Outros Estados & 47 & 13,5 & \\
& Sim & 40 & 11,5 & 349 \\
& Não & 309 & 88,5 & \\
\hline
\end{tabular}

* respostas válidas, excluídas as respostas ignoradas

$45,6 \%$ do sexo feminino; $57,2 \%$ eram casados e $21,8 \%$ solteiros. Com relação à naturalidade, 52\% eram de Salvador, 34,5\% do interior do estado da Bahia e 13,5\% de outros Estados. Linhagem médica (ter pai e/ou mãe médicos) foi relatada por 11,5\% dos médicos estudados (Tabela I).

A idade média da amostra de médicos estudada foi de 45,2 — 13,6 anos. Os homens apresentaram idade média de 48, I \pm I4,9 anos e as mulheres, 4l,6 \pm 10,8 anos. Com relação ao tempo de trabalho, os médicos apresentavam em média 24,2 \pm 18,6 anos de trabalho na área e as médicas, 18,0 \pm 14,7 anos. Na faixa etária menor que 45 anos, 0 sexo feminino representava 55,4\% ( I02) da amostra estudada.

A Tabela 2 apresenta algumas características da formação técnico-profissional dos médicos de Salvador. Programa de residência médica concluído foi referido por $67,5 \%$ dos médicos. Título de Especialista foi referido por $67,9 \%$ dos profissionais. Cursos de mestrado e doutorado foram realizados por $10,4 \%$ e $5,2 \%$ dos médicos estudados, respectivamente. Programa de residência médica foi realizado em instituição pública por $86,1 \%$ dos médicos e, em instituição privada, por 13,9\%. Em relação ao local de realização do programa de residência médica, 79\% o fizeram no Estado da Bahia, e 99, I\% no Brasil. Dentre os 317 médicos que responderam à questão, 87 referiram não ter especialidade. Dentre as especialidades médicas, destacaram-se como as cinco mais freqüentes: Ginecologia e obstetrícia (12,6\%), Pediatria (8,2\%), Cirurgia Geral (5,7\%), Oftalmologia (5,0\%) e Medicina Interna (4,4\%). Quanto ao tempo de graduação, 39,3\% dos médicos apresentavam menos de 15 anos de graduados e apenas $16 \%$ apresentavam mais de 30 anos de graduados.

Trabalho ativo atual como médico foi referido por $83,6 \% \mathrm{da}$ amostra; 13,5\% estavam ativos em uma atividade e aposentado em outra. Dessa forma, 97, 1\% dos médicos que responderam ao questionário estavam em atividade, 2,3\% eram aposentados e $0,6 \%$ abandonaram a profissão. Entre os médicos estudados, 25,9\% desenvolviam atividade de trabalho em outros municípios. Entre os profissionais estudados, $86,2 \%$ tinham a medicina como única fonte de renda, $72,3 \%$ trabalhavam no setor privado, 71,1\% afirmaram desenvolver atividade em consultório, 


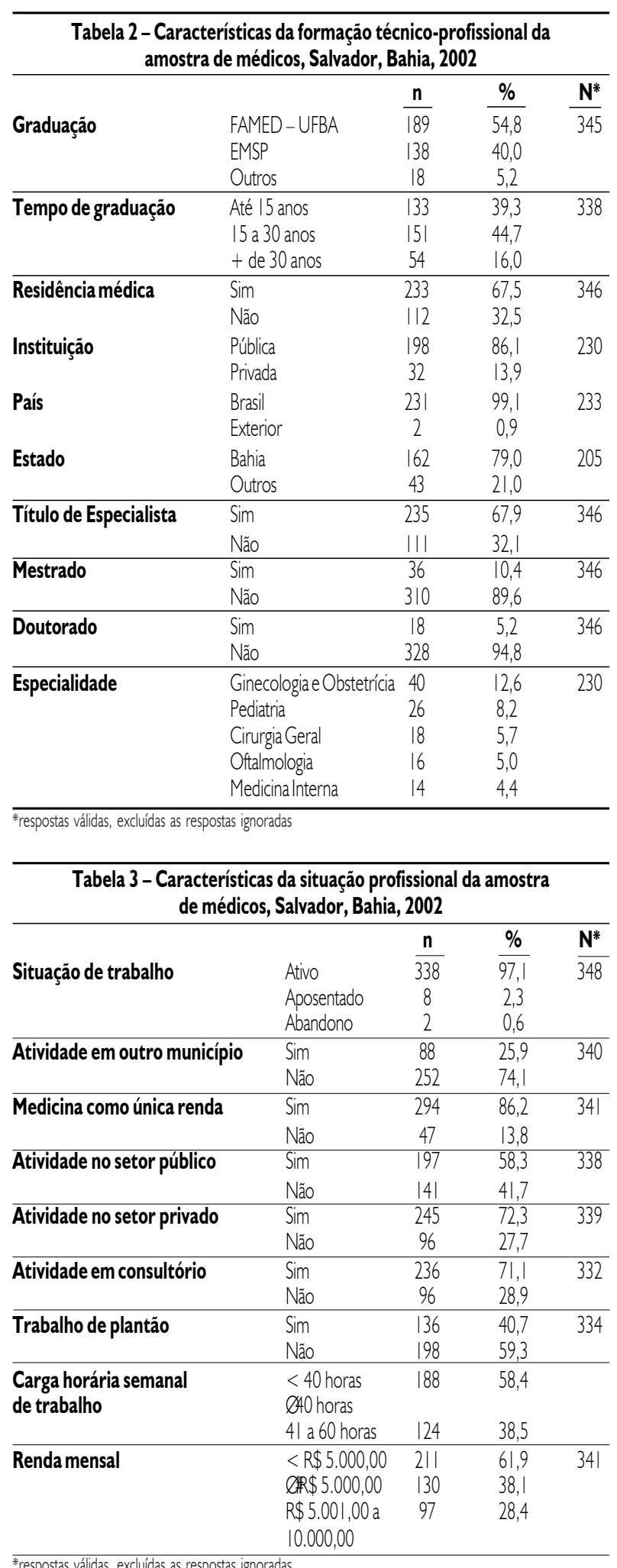

*respostas válidas, excluídas as respostas ignoradas
58,3\% trabalhavam no setor público e 40,7\% trabalhavam em regime de plantão. Carga horária semanal de trabalho como plantonista igual ou superior a 24 horas foi referida por 67 médicos, correspondendo a $20 \%$ da amostra com respostas válidas. Entre os médicos estudados, 58,4\% apresentavam carga horária semanal de trabalho médico igual ou superior a 40 horas. Destes, 38,5\% apontaram 41 a 60 horas de trabalho semanal. A renda mensal aproximada obtida com 0 trabalho médico foi inferior a $R \$ 5.000,00$ para $61,9 \%$ dos médicos (Tabela 3).

Os médicos apontaram como sendo o principal local de sua prática profissional o setor privado (38\%), o setor público $(35,6 \%)$ e o consultório paricular $(26,7 \%)$. O número de inserções no mercado de trabalho foi de uma (21\%), duas $(39,2 \%)$, três $(28,4 \%)$ inserções, até o máximo de seis inserções observado para um caso. A média foi de duas inserções de trabalho por médico. Portanto, $79 \%$ dos médicos apresentavam formas mistas de inserção ao mercado, distribuídas segundo: $11,7 \%$ consultório particular típico (pagamento pelo serviço realizado diretamente pelo paciente), 24,4\% por assalariamento no setor privado, 41,9\% por prestação de serviço (remuneração por procedimento realizado), 43,4\% por meio de consultório particular atííco (pagamento pelo serviço realizado por operadoras de serviços de saúde) e 47,6\% por assalariamento no setor público. Formas puras de inserção foram encontradas para 70 médicos: empresário (0,9\%), consultório particular típico (I,5\%), consultório particular atípico $(1,8 \%)$, assalariado do setor privado $(2,7 \%)$, assalariado no setor público $(8,7 \%)$ e prestação de serviço (5,4\%) (Tabela 4).

Verificou-se que, entre os médicos que trabalhavam no setor público, $86,9 \%$ eram assalariados e, entre os que trabalhavam no setor privado, $36,3 \%$. Estas proporções permitem avaliar a magnitude do trabalho informal nos setores público e privado da medicina em Salvador.

Entre as queixas de saúde, destacaram-se como as mais freqüentes cansaço mental (54,1\%), dor nas costas (43,3\%), sonolência $(37,5 \%)$, dor nas pernas $(37,2 \%)$, dor nos braços $(31,3 \%)$, esquecimento $(30,8 \%)$, nervosismo $(28,4 \%)$ e insônia $(25,1 \%)$. Dentre os diagnósticos referidos desde que trabalhavam como médicos, destacaram-se: varizes em membros inferiores (89,4\%), lombalgia (54,2\%), hipertensão arterial (24\%), infecção urinária (21,1\%) e sinusite (15,2\%) (Tabela 4). Com exceção da hipertensão arterial, as médicas apresentaram frequêencias mais elevadas, tanto das queixas de saúde como dos diagnósticos referidos.

A prevalência de distúrbio psíquico menor (DPM) foi de 26\%. Entre as mulheres, a prevalência foi de $34,6 \%$ e entre os homens, de $17,8 \%$, sendo esta diferença estatisticamente significante $(p<0,05)$. $O$ uso de bebida alcoólica foi referido por $48,6 \%$ da amostra; o teste Cage triou 20 (5,8\%) indivíduos como bebedores-problema. A prevalência de positividade ao Cage entre os médicos foi de 6,4\% e entre as médicas foi de $4,3 \%(p>0,05)$. 


\begin{tabular}{|c|c|c|c|c|}
\hline \multicolumn{5}{|c|}{$\begin{array}{l}\text { Tabela } 4 \text { - Características da situação profissional da amostra } \\
\text { de médicos, Salvador, Bahia, } 2002\end{array}$} \\
\hline & & $\mathrm{n}$ & $\%$ & $\underline{\mathbf{N}^{*}}$ \\
\hline Setor de atividade principal & $\begin{array}{l}\text { Público } \\
\text { Privado } \\
\text { Consultório }\end{array}$ & $\begin{array}{c}119 \\
127 \\
88 \\
\end{array}$ & $\begin{array}{l}35,6 \\
38,0 \\
26,4 \\
\end{array}$ & 334 \\
\hline $\begin{array}{l}\text { Número de inserções no } \\
\text { mercado de trabalho }\end{array}$ & $\begin{array}{l}\text { l inserçãão } \\
2 \text { inserções } \\
3 \text { inserções } \\
4 \text { inserções } \\
5 \text { inserções } \\
6 \text { inserções }\end{array}$ & $\begin{array}{c}70 \\
13 \mid \\
95 \\
32 \\
7 \\
1\end{array}$ & $\begin{array}{l}21,0 \\
39,2 \\
28,4 \\
9,5 \\
2,0 \\
0,3\end{array}$ & $\begin{array}{l}334 \\
334 \\
334 \\
334 \\
334 \\
334\end{array}$ \\
\hline $\begin{array}{l}\text { Formas mistas de inserção } \\
\text { no mercado de trabalho** }\end{array}$ & $\begin{array}{l}\text { Consultório típico } \\
\text { Consultório atíicico } \\
\text { Empresário } \\
\text { Assalariado setor privado } \\
\text { Assalariado setor público } \\
\text { Prestação de serviço }\end{array}$ & $\begin{array}{l}39 \\
144 \\
143 \\
81 \\
159 \\
139\end{array}$ & $\begin{array}{l}11,7 \\
43,4 \\
41,8 \\
24,4 \\
47,6 \\
41,9\end{array}$ & $\begin{array}{l}332 \\
332 \\
342 \\
332 \\
334 \\
332\end{array}$ \\
\hline $\begin{array}{l}\text { Formas puras de inserção } \\
\text { no mercado de trabalho*** }\end{array}$ & $\begin{array}{l}\text { Consultório típico } \\
\text { Consultório atíico } \\
\text { Empresário } \\
\text { Assalariado setor privado } \\
\text { Assalariado setor público } \\
\text { Prestação de serviço }\end{array}$ & $\begin{array}{l}5 \\
6 \\
3 \\
9 \\
29 \\
18\end{array}$ & $\begin{array}{l}1,5 \\
1,8 \\
0,9 \\
2,7 \\
8,7 \\
5,4\end{array}$ & $\begin{array}{l}332 \\
332 \\
342 \\
332 \\
334 \\
332\end{array}$ \\
\hline
\end{tabular}

*respostas válidas, excluídas as respostas ignoradas

**cada opção de inserção ao mercado foi tomada como variável isolada, logo o total obtido não equivale a $100 \%$

**** as formas puras de inserção foram excluídas das mistas

\begin{tabular}{lccc}
\hline \multicolumn{4}{c}{ Tabela 5 - Características da situação de saúde da amostra } \\
de médicos, Salvador, Bahia, 2002
\end{tabular}

* respostas válidas, excluídas as respostas ignoradas

\section{Discussão}

Os resultados indicam que a medicina em Salvador é exercida principalmente por profissionais jovens e do sexo masculino. Entretanto, uma mudança pode estar em andamento, pois constatou-se elevada participação feminina na categoria médica na faixa etária menor que 45 anos. Esse resultado indica que, em poucos anos, o sexo feminino poderá predominar na categoria médica na cidade de Salvador. Esses resultados assemelham-se aos obtidos em outros estudos realizados no Brasili,4.

Os resultados apontaram uma forte adesão profissional dos médicos de Salvador, caracterizada pela baixa frequêencia de abandono da profissão. Observou-se também a inexistência de médicos sem ocupação no mercado de trabalho. A medicina ainda é uma atividade profissional que garante trabalho e o sustento dos seus participantes, no tocante à remuneração. Comprovou-se, ainda, que existe em Salvador um amplo e diversificado mercado de trabalho, representado pelo setor privado, atividades em consultório e pelo setor público. Esses resultados assemelham-se aos obtidos em outros estudos ${ }^{3,4}$.

Os médicos de Salvador apresentaram elevada frequêencia de pósgraduação sob a forma de programas de residência médica. Esse resultado associou-se à elevada frequêencia de médicos com título de especialização. As formas de pós-graduação stricto sensu (mestrado e doutorado) são pouco realizadas pelos membros da categoria. Esse resultado talvez esteja associado a uma maior valorização no mercado de trabalho dos programas de residência médica, que se caracterizam como treinamento em serviço, nas diversas especialidades ${ }^{3,4}$.

Os resultados apontaram uma sobrecarga de trabalho entre os médicos de Salvador, especialmente nas atividades de plantão. Indicam também excesso de inserções de trabalho. Esses resultados são semeIhantes aos obtidos em outros estudos ${ }^{2,3,4,5}$. Entretanto, esta situação apresenta-se inadequada, pois a atividade médica necessita de tempo para interação entre médico e paciente, acompanhamento e estudo, bem como para atualização científico-tecnológica.

Observada isoladamente, a remuneração obtida com o trabalho médico pode ser considerada satisfatória. Entretanto, observou-se que, para obtenção desse resultado financeiro, faz-se necessária uma sobrecarga de trabalho, gerando uma baixa remuneração por hora trabalhada. Esses resultados assemelham-se ao de outros estudos ${ }^{3,4}$.

Os resultados indicaram que o consultório permanece como uma forma de inserção no mercado de trabalho. Entretanto, o consultório típico apresentou baixa freqüência, nos médicos de Salvador, tanto na forma pura como na forma mista. Como em outros estudos, os resultados apontaram para o crescimento do trabalho informal, por procedimento, este relacionado à subcontratação, terceirização e precarização do trabalho médico, no setor público, mas principalmente no setor privado, com redução do trabalho assalariado 3, $3,33,34,35$.

Entre os problemas de saúde, destacaram-se as queixas e diagnósticos referentes a transtornos mentais e posturais, que podem estar associados às características tradicionais e atuais do trabalho médico: atender a pacientes, enfrentar a dor, o sofrimento a morte, excesso de trabalho, especialmente em atividade de plantão, baixa remuneração e instabilidade no trabalho ${ }^{29}$.

Os médicos apresentaram prevalência de DPM mais elevada que a de outras categorias profissionais, como siderúrgicos ${ }^{25}$, agricultores ${ }^{26}$, trabalhadores da indústria de papel e celulose ${ }^{27}$, metroviários ${ }^{28} \mathrm{e}$ professores de nível médio ${ }^{36,37}$ e universitário ${ }^{38}$. 
A existência, no trabalho médico, de condições desencadeadoras de sofrimento, estresse e ansiedade é uma realidade não mais contestada. No entanto, as repercussões sobre a saúde dos trabalhadores médicos, reconhecidamente expostos a fatores estressantes e ansiogênicos, ainda são pouco conhecidas. A organização do trabalho é o principal orientador da vida mental do trabalhador. A partir dos elementos presentes na organização do trabalho, deve-se investigar o que, especificamente, pode ser tomado como fonte de sofrimento e de danos à saúde dos médicos ${ }^{12,39,40}$.

Quando é possível definir os próprios ritmos e formas de executar as tarefas, personalizando o processo de trabalho, o que predominantemente entra em jogo é a criatividade e a inventividade. Esse tipo de comportamento é denominado livre. Esse comportamento transforma e reordena o trabalho na direção do prazer, quando possibilita um ajuste entre as exigências do processo de trabalho e a estrutura psíquica de cada um, abrindo brechas para a transformação da realidade segundo os próprios desejos. Esse padrão operacional seria típico da medicina artesanal| ${ }^{\mid 2,39,40,41,42,43}$

Quando ocorre a impossibilidade de atendimento dessas condições básicas, por meio de processos de trabalho que atuam no sentido de bloquear o comportamento livre, ocorre uma mudança no significado do trabalho dos indivíduos. Ou seja, na medida em que o processo de trabalho é conduzido em direção oposta ao comportamento livre, adotando modos operatórios rígidos e padronizados, menos o trabalho representará para o indivíduo a possibilidade de equilíbrio mental e mais próximo estará de constituir fonte de sofrimento. Esse padrão operacional seria típico do modelo taylorista/fordista de organização do trabalho ${ }^{39,40,44}$. A transformação do trabalho médico de um padrão artesanal (medicina liberal) para um padrão taylorista/fordista não deve ter ocorrido sem sofrimento psíquico dos médicos. Mais recentemente, transformações decorrentes da implementação do novo padrão de organização do trabalho (modelo japonês) podem ser apontadas como possíveis fatores de risco à saúde mental dos médicos. Este modelo trouxe rápidas transformações, instabilidade na contratação, consolidação da remuneração variável (por procedimento) e rígido controle externo (controle de qualidade) ${ }^{45}$.

Neste contexto econômico-social, fica evidente a dificuldade de estudar o trabalho médico, com todas as suas particularidades e heterogeneidade estrutural.

Deve-se tecer algumas considerações metodológicas. Esse estudo apresentou uma grande proporção de perdas e recusas (57,3\%), que poderia ter comprometido a generalização dos resultados. Estudos de amostras aleatórias de médicos que adotaram a estratégia de envio a domićlio do questionário apresentaram proporções de devolução semeIhantes à obtida nesta pesquisa. No estudo "Perfil dos médicos no Brasil", a frequêencia de perdas e recusas atingiu 48,2\% para o País, 45, I \% para a Bahia e $46 \%$ para Salvador. Um inquérito sobre a atenção dos médicos em relação à sua própria saúde, realizado em Barcelona, Espanha, obteve $66 \%$ de perdas e recusas ${ }^{3,46}$. Esses resultados apontam a grande dificuldade na obtenção de respostas a pesquisas com médicos.

Dados obtidos junto ao Conselho Regional de Medicina do Estado da Bahia demonstram que 52,6\% dos médicos de Salvador pertencem ao sexo masculino e $47,4 \%$ ao sexo feminino. A freqüência das especialidades Ginecologia e Obstetrícia (10,0\%), Pediatria (9,2\%),
Cirurgia Geral (7,6\%), Oftalmologia (4,9\%) e Medicina Interna (7,2\%) foram semelhantes às obtidas no nosso estudo.

O viés de seleção pode ocorrer devido a diferenças sistemáticas entre aqueles que foram selecionados para o estudo e aqueles que não o foram ${ }^{47}$. Neste estudo, isso aconteceria se um ou mais grupos que compõem a população não estivessem devidamente representados na amostra selecionada para a pesquisa. Os resultados sugerem que esse viés foi minimizado. Entretanto, a análise estatística de certas variáveis de baixa freqüência, como algumas especialidades médicas, foi prejudicada em decorrência da pouca expressividade da amostra.

\section{Conclusão}

Os resultados apresentados refletem as transformações ocorridas na base técnica e na produção do trabalho médico na sociedade brasileira, e em Salvador, especificamente. Nesta capital, fica evidente que a medicina passou de um padrão artesanal para uma nova e complexa modalidade de organização do trabalho. 0 cenário exposto apresenta-se bastante desfavorável aos médicos de Salvador devido a: sobrecarga de trabalho, especialmente em atividades de plantão; condições de trabalho precárias, regidas preferencialmente pela remuneração por procedimento, especialmente no setor privado; baixa remuneração e elevada freqüência de queixas físicas e psíquicas. Esse cenário desfavorável aos médicos pode refletir-se na sociedade, por meio da baixa qualidade do atendimento prestado aos usuários dos serviços de saúde. Esses resultados podem explicar o descontentamento dos médicos e da sociedade em geral com os serviços de saúde e com a atenção médica. As condições de trabalho e saúde observadas apontam para a necessidade de mudanças profundas na organização do trabalho médico.

Conflito de interesse: não há.

\section{SUMMARY \\ Conditions of work and health of physicians in the City of Salvador, Brasil}

BACKGROUND. The conditions of work and health of physicians in the city of Salvador, State of Bahia, Brasil were described.

Methods. A cross sectional study investigated a random sample of 350 physicians. Information about the physical and mental health, living habits and working conditions of these physicians was collected by self-reported, individual questionnaires forwarded by mail.

RESULTS. The physicians reported excessive workloads, more than one job, low income, and precarious contracting with payment for each procedure performed. A private practice was also reported by $45.2 \%$ of the physicians however only $1.5 \%$ exercised it exclusively. The most frequent health complaints were related to body posture and mental health.

CONCLUSION. The prevalence of minor psychological disorders was of 26.0\%. The observed conditions of work and health point to a need for major changes in the organization of medical work. [Rev Assoc Med Bras 2006; 52(2): 97-102]

KEY WORDS: Work. Health. Physicians. 


\section{REFERÊNCIAS}

I. Brasil. Ministério da Saúde. Trabalhadores em Saúde. Rio de Janeiro: FIOCRUZ/ENSP; 1998.

2. Conselho Federal de Medicina. Os Médicos e a Saúde no Brasil. Brasilia,DF: CFM; 1998.

3. Machado $\mathrm{MH}$. Os Médicos no Brasil: um retrato da realidade. Rio de Janeiro: Fiocruz; 1997

4. Conselho Federal de Medicina. Os Médicos e o Seu Trabalho. Brasília, DF: CFM; 2004.

5. Conselho Regional de Medicina do Estado de São Paulo (CREMESP). Mercado de Trabalho Médico no Estado de São Paulo. São Paulo: CREMESP; 2002.

6. Marques ACPR e Alves HNP. O uso de álcool e outras substâncias com potencial de abuso. In: SIMESP (org) Desgaste Físico e Mental do Cotidiano Médico. São Paulo: SIMESP; 2002.

7. Martins LA. Morbidade psicológica e psiquiátrica na população médica. Bol Psiq, 1990; 22-23: 9-15.

8. Martins LA. Atividade médica: fatores de risco para a saúde mental do médico. Rev Bras Clin Terap, 1991; 20(9): 355-64.

9. Martins LA. Vicissitudes do exercício da medicina e saúde psicológica do médico. Arq Bras Edocrinol Metab, 1995; 39: 188-93.

10. Martins LA. Morbidade psicológica e psiquiátrica entre médicos. In: Agosto FM, Peixoto R e Bordin, R (org). Riscos da Prática Médica. Porto Alegre: Da Casa Editora; 1998.

I I. Moura MA, Peixoto R, Agosto FM. A saúde ocupacional dos médicos. In: Agosto FM,Peixoto R e Bordin, R (org), Riscos da Prática Médica. Porto Alegre: Da Casa Editora; 1998.

12. Nascimento Sobrinho CL e Nascimento MA Trabalho e Saúde dos Médicos. In: SIMESP (org) Desgaste Físico e Mental do Cotidiano Médico. São Paulo: SIMESP; 2002.

13. Colford JM e Litt RC. The revealled sleeve of care - managing the stress of residency training. JAMA, 1989; 261; 889-93.

14. Maccue JD. The effects of stress on physicians and their medical practice. N Engl J Méd, 1982; 306: 485.

15. Mawardi BH. Satisfactions, dissatisfactions and causes of stress in medical practice. JAMA, 1979; 241: 1483-6.

16. Pilowski L, O’Sullivan G. Mental illness in doctors. BMJ, 1989; 298: 26970.

17. Ross M. Suicide among physicians - a psychological study. Dis Nerv Syst, 1973:31: | 45-50.

18. Sociedade Brasileira de Anestesiologia. Dependência química entre anestesistas. São Paulo: SBA; 2002

19. Ullmann D, Phillips RL, Beeson WL, Dewey HG, Brin BN, Kuzma JW, Mathews CP, Hirst AE. Cause-specific mortality among physicians with differing life-styles. JAMA, 199|; 265( | 8): 2352-9.

20. Lwanga SK e Lemeshow S. Simple size determination in health studies. A pratical manual. Geneva: WHO; 1991.

21. Gomes MM. Medicina Baseada em Evidências. Rio de Janeiro: Reichmann e Affonso Editores; 200I.

22. Kirkwood BR. Essentials of medical statistics. London: Blackwell Scientific Publications; 1988.

23. Mari JJ. Minor psychiatric morbidity in three primary medical care clinics in the city of São Paulo. (Tese de doutorado). University of London / Institute of Psychiatric: Londres; 1986.

24. Mari JJ, Williams P. Validity study of psychiatric screening questionnaire (SRQ-20) in primary care in the city of São Paulo. Brit J Psych, 1986; I48: 23-36.

25. Borges LH, Faria MAM. Transtornos mentais menores entre trabalhadores de uma usina siderúrgica. Revista Brasileira de Saúde Ocupacional, 1993; 21: 7-18.

26. Faria NMX, Facchini LA, Fassa AG, Tomasi E. Processo de produção rural e saúde na Serra Gaúcha. Revista de Saúde Pública, 1999; 33: 391-400.
27. Fassa AG, Facchini LA, Dall'Agnol MM. Trabalho e morbidade comum em indústria de celulose e papel: um perfil do segundo setor. Cadernos de Saúde Pública, 1996; 12: 297-307.

28. Jardim RS, Perecmanis L, Silva Filho JF. Processo de trabalho e sofrimento psíquico: o caso dos pilotos de metrô do Rio de Janeiro - II. J Bras Psiq, 1996; 45: 323-333.

29. Pitta A. Hospital, dor e morte como ofício. São Paulo: Editora Hucitec; 1990.

30. Masur J e Monteiro MG. Validation of the "CAGE" alcoholism screening test in a brasilian psychiatric inpatient hospital setting. Braz J Med Biol Res, 1983; 16:215-218.

31. Brasil. Ministério da Saúde. Conselho Nacional de Saúde. Diretrizes e normas regulamentadoras de pesquisas envolvendo seres humanos: resolução 196/96, do Conselho Nacional de Saúde. Rio de Janeiro: FIOCRUZ; 1998.

32. SPSS INC. SPSS Base 9.0 - Applications Guide. Chicago, EUA; I991.

33. Machado $\mathrm{MH}$. Gestão do Trabalho em saúde no contexto de mudanças. RAP, 2000; 34: 133-146.

34. Nascimento Sobrinho CL e Formigli VLA. Organização, Financiamento e Gestão dos Serviços de Saúde no Brasil. Texto Didático da disciplina MED209 - Introdução à Medicina Social, Salvador, Departamento de Medicina Preventiva, Universidade Federal da Bahia; 2001.

35. Donnangello MCF. Medicina e Sociedade. O Médico e o seu mercado de trabalho. São Paulo: Pioneira; 1975.

36. Silvany Neto AM, Araújo TM, Kawalkievicz C, Dutra FRD, Azi GR, Alves $\mathrm{RL}$, Reis EJFB. Condições de Trabalho e saúde dos professores da rede particular de ensino, Salvador, Bahia. Revista Baiana de Saúde pública, 2000; 24: 42-56.

37. Silvany Neto AM, Araújo, TM, Kawalkievicz C, Lima BGC, Dutra FRD, Paiva LC, Sarno MM. Condições de Trabalho e saúde em professores da rede particular de ensino, na Bahia. Rev Bras de Saúde Ocup, 1998; 24: | 15-24.

38. Wernick R. Condições de Saúde e Trabalho dos Docentes da Universidade Federal da Bahia, Salvador, Bahia. Dissertação de Mestrado, apresentada ao Programa de Pós-graduação em Saúde Coletiva, da Universidade Federal da Bahia - UFBA; 1999.

39. Dejours C. A loucura do Trabalho: Estudo de Psicopatologia do Trabalho. SãoPaulo: Editora Cortez-Oboré; 1987.

40. Dejours C. Introdução à Psicopatologia do Trabalho. Tempo Social - Rev Sociol USP, 1989; I(2): 97-103.

41. Mendes Gonçalves RB. Medicina e História. Raízes Sociais do Trabalho Médico. (Dissertação de Mestrado) Universidade de São Paulo: São Paulo; 1979.

42. Schraiber LB. O Trabalho Médico e a Clínica na Medicina Moderna. Centro de Formação dos Trabalhadores da Saúde. São Paulo; I 992. p. I 41

43. Schraiber LB. O Médico e o seu Trabalho. Limites da Liberdade. São Paulo: Editora HUCITEC; 1993.

44. Schraiber LB. Profesión Médica representación, trabajo y cambio. Educación Médica y salud, 1991; 25(I): 58-7I.

45. Druck MG. Terceirização: (des)fordizando a fábrica. Salvador: EDUFBA; 1999.

46. Bruguera M, Gurí J, Arteman A, Valldosera JG, Carbonell J. La atención de los médicos hacia el cuidado de su própria salud. Resultados de uma encuesta postal. Méd Clin, 2001; I 17(3); 492-4.

47. Pereira MG. Epidemiologia Teoria e Prática. Rio de Janeiro: Guanabara Koogan; 1995. 